\title{
METHOXYFLURANE: AGENT D'INDUCTION EN ANESTHESIE PEDIATRIQUE
}

\author{
J. Maurice Bergeron, M.D., et Jean Ménard, M.d. ${ }^{\circ}$
}

LA DÉCOuverTe du méthoxyflurane (2,2 dichloro 1,1 difluoro éthyl méthyl éther), substance anesthésique volatile tenant à la fois des halogénés et des éthers, a surement fourni du travail à bien des chercheurs qui ont voulu découvrir tous ses secrets.

Les avantages du méthoxyflurane sont désormais reconnus et il est indéniable que cette substance est considérée actuellement comme agent de maintien de premier ordre. Moins connu toutefois est son rôle comme seul agent d'induction anesthésique, surtout chez l'enfant. Le méthoxyflurane est certainement le plus puissant des agents anesthésiques par inhalation à cause de sa solubilité dans les graisses. Toutefois sa lenteur d'action est due à sa basse pression de vapeur à la température ambiante, à son coefficient de solubilité air, sang et sa solubilité dans les tissus tampons.

Ce qui semble donc être la pierre d'achoppement du méthoxyflurane est la rareté des molécules gazeuses disponibles pour l'établissement des coefficients air/sang/cerveau nécessaires à l'apparition rapide du sommeil. Cela peut-être corrigé de façon satisfaisante. L'apport total des molécules de substance anesthésique disponibles peut-être influencé de deux façons:

1. Par l'utilisation d'un vaporisateur puissant capable de fournir une concentration adéquate (maximum possible de $3.3 \%$ à $20^{\circ} \mathrm{C}$.) .

2. Par le passage dans le vaporisateur d'un débit gazeux élevé qui augmentera la quantité de méthoxyflurane vaporisée mais non la concentration. L'augmentation du volume minute et conséquemment l'augmentation du gradient de pression alvéoles-sang, favorise la solubilité du méthoxyflurane dans le sang.

Voici un rapport clinique sur l'utilisation du méthoxyflurane comme agent d'induction dans 250 anesthésies chez des enfants de un à douze ans, la moyenne d'âge se situant vers six à sept ans. Tous étaient de bons risques anesthésiques et beaucoup de ces patients furent admis à l'hôpital le matin de l'intervention et le quittèrent le jour même. Ils furent soumis soit à une amygdalectomie, une herniotomie, une appendicectomie ou soit à une extraction dentaire.

\section{Prémédication}

Les rôles attribués et exigés de la prémédication ont beaucoup évolué avec l'arrivée sur le marché des anesthésiques modernes et surtout des halogénés. Diminuer l'anxiété et l'appréhension, occasionner la somnolence sans dépression cardio-vasculaire ou respiratoire, sont les objectifs poursuivis en premier lieu. L'usage d'un barbiturique est parfaitement adapté à ce besoin tandis qu'il semble

${ }^{\circ}$ Hôpital Sainte-Thérèse, Shawinigan, P.Q. 
préférable de réserver le sédatif narcotique au seul patient souffrant avant l'anesthésie.

Le méthoxyflurane n'a pas tendance à modifier le rythme sinusal normal et il ne favorise que très peu la sécrétion salivaire et muqueuse. L'usage d'un dérivé belladoné reste certes encore un adjuvant utile mais sa nécessité n'est plus impérieuse.

Les associations pentothal-rectal-atropine et démerol-atropine furent employées à dose conservatrice et il faut admettre que la première nous a procuré des patients mieux préparés, plus calmes et plus coopératifs. Quelques cas (10) furent également anesthésiés sans aucune prémédication avec des résultats satisfaisants.

\section{MÉTHODES D’AdMINISTRATION}

Trois techniques différentes d'anesthésie furent utilisées: (a) une technique avec rebreathing partiel sans absorption de $\mathrm{CO}_{2},(b)$ une technique sans rebreathing, $(c)$ le goutte à goutte. Excepté pour quelques cas (pour les besoins de l'expérience), un seul type de vaporisateur fut employé: le pentec à concentration maximum de 1.5 pour cent qui s'est avéré efficace. De fait, il n'est pas nécessaire de recourir à un vaporisateur plus puissant.

Méthode avec rebreathing partiel sans absorption de $\mathrm{CO}_{2}$, et méthode sans rebreathing.

Les données de ces deux méthodes sont décrites simultanément puisqu’elles sont pratiquement semblables:

1. Le set "MIE" fut utilisé pour obtenir le rebreathing partiel sans absorption de $\mathrm{CO}_{2}$.

2. Des valves unidirectionnelles classiques ont servi au montage de la technique sans rebreathing.

Un débit de 4 à 8 litres suivant le poids fut utilisé, formé de $\mathrm{N}_{2} \mathrm{O}$ et d'O $\mathrm{O}_{2}$ à parties égales. L'introduction des vapeurs de méthoxyflurane dans le mélange gazeux inspiré fut graduelle mais aussi rapide que le permettait la tolérance du patient: la concentration maximale de 1.5 pour cent étant atteinte dans la première minute. Cette façon de procéder est bien tolérée si l'on se base sur la présence exceptionnelle de toux et la perte de réponse à la parole survient rapidement après 90 ou 120 secondes d'inhalation.

La phase d'excitation est quelquefois absente: le plus souvent, elle se manifeste sobrement par une courte retenue de la respiration accompagnée de faibles mouvements de membres et du tronc ne nécessitant pas de rétention forcée quoique son intensité semble liée à la qualité de la prémédication.

Une respiration rythmique rapide mais dont l'amplitude est encore normale apparait après $2 \gamma_{2}^{\prime}$ à 3 minutes d'inhalation du méthoxyflurane à 1.5 pour cent. Par suite, l'appronfondissement de l'anesthésie est progressif ne s'accompagnant que d'une légère stimulation salivaire ou muqueuse; aucun spasme laryngobronchique, aucun vomissement.

Les signes classiques de l'évolution anesthésique sont absents. Des signes plus 
subtils doivent être surveillés; la chute généralement légère de la T.A., le ralentissement et la petitesse du pouls, la perte d'amplitude de la respiration qui a tendance à demeurer longtemps rapide.

Vers la $5^{\mathrm{e}}$ ou $6^{\mathrm{e}}$ minute, les pupilles sont centrées et le relâchement des maxillaires est adéquat et permet l'introduction facile du laryngoscope. Même si lintubation est possible à ce stage due à la béance des cordes vocales, elle demeure une procédure trop hâtive qui déclanche des réactions réflexes respiratoires et cardiovasculaires: toux, bucking, respiration retenue, accélération du pouls et hausse de la T.A. qui restent toutefois facilement contrôlables par quelques pressions manuelles du ballonnet. Toutefois, à ce stage, cette manœuvre est trop précoce et peut théoriquement être source de troubles graves.

Une durée d'inhalation de 7 à 8 minutes, en respiration spontanée semble nécessaire pour une technique d'intubation sans myorésolutif qui ne déclanche pas de réflexe respiratoire ou cardiovasculaire. A ce stage, la respiration voit son amplitude amputée d'environ 30 pour cent (mesurée avec ventimètre de Wright) quoique son rythme soit rapide; le chiffre de la T.A. a parfois chuté de 15 à 25 $\mathrm{mm}$. Hg; le pouls s'est fait plus lent et plus petit; la pression digitale du bouquet de Riolan a cessé d'être réllexogène. Toutefois, l'incision de la peau à ce stage amène encore une réponse appréciable car il faut se souvenir que la lenteur d'établissement de l'analgésie cutanée est l'apanage des halogénés fluorés moderne. ${ }^{1}$

Le système cardio-vasculaire, au cours de l'induction avec le méthoxyflurane en respiration spontanée est particulièrement stable. Il survient bien une légère chute de T.A. et un minime ralentissement du pouls mais ces manifestation devraient-elles devenir plus marquées qu'elles ont l'avantage d'être facilement et rapidement corrigées par l'arrêt de l'inhalation du méthoxyflurane. L'arythmie cardiaque est exceptionnelle; un cas de "wandering pacemaker" fut observé chez un patient. Cette arythmie, lorsque présente signe à coup sûr un début de surdosage anesthésique et il suffit d'alléger la profondeur de l'anesthésie pour assister rapidement au retour d'un rythme sinusal normal. Des travaux antérieurs ont permis d'établir l'effet dépresseur du méthoxyflurane sur le myocarde qui amène baisse du débit cardiaque et vasoconstriction périphérique compensatrice. ${ }^{2}$ Ces manifestations sont proportionnelles à la profondeur de l'anesthésie et l'apparition d'une pâleur extrême souvent accompagnée de cyanose des lèvres et de la région sous-unguéale signe encore un surdosage d'anesthésique. Aucune pâleur de ce genre n'a été observée ni pendant ni après l'anesthésie chez aucun des cas de cette série, mais elle est alors facilement corrigible par l'usage de l'isuprel. Par suite de son action sélective sur les récepteurs adrénergiques $\beta$, l'isuprel a une influence positive sur la chronotropicité et l'intropicité du muscle cardiaque et son action sur la résistance périphérique totale en est une de dilatation (dilatation des lits vasculaires rénaux, fémoraux, mésentériques et coronaires).$^{3}$ Ses actions sont donc à l'inverse de celles du méthoxyflurane sur le cœur et la circulation périphérique.

L'influence du méthoxyflurane sur la respiration se résume à une baisse progressive de l'amplitude respiratoire et à une tendance légère à la tachypnée de sorte que le minute-volume, mesuré au moyen du ventimètre de Wright, montrait 
une diminution moyenne de l'ordre de 30 pour cent par rapport au chiffre théorique normal, au moment de l'intubation. Le méthoxyflurane est une substance anesthésique très peu irritante pour les voies respiratoires.

$\mathrm{Au}$ cours des anesthésies (10) où aucune prémédication ne fut employée, les changements suivants furent observés:

1. La période d'excitation fut plus marquée quoique son intensité en fut encore modérée.

2. Le temps d'inhalation du méthoxyflurane nécessaire à l'obtention d'un niveau anesthésique identique fut prolongé d'environ 20 pour cent.

3. La tendance aux modifications cardio-vasculaires (baisse de T.A., relentissement et petitesse du pouls) est plus marquée surtout si l'anesthésie est poussée à des niveaux profonds: elle demeure toutefois facilement réversible par l'arrêt d'administration de la substance. Aucune anomalie clinique ou électrique ne fut observée à l'E.C.G.

4. Une tendance plus marquée à la tachypnée semble probable.

5. Aucune manifestation d'hypersécrétion salivaire ou muqueuse n'a été observée. Il semble donc que le rôle de la prémédication perd beaucoup de son impératif de protection cardiaque et d'asséchement des sécrétions devant une substance telle que le penthrane. Son utilité se résume à la tranquilisation du sujet.

Ces résultats obtenus avec linhalation du méthoxyflurane à la concentration de 1.5 pour cent paraissent satisfaisants au point de vue temps et sécurité. Il reste toutefois possible de jouer sur le facteur temps, mais il faut dire que ces deux facteurs varient dans une direction inverse: ce que l'on gagne en rapidité d'induction, on le perd en sécurité d'emploi. Quels moyens sont possibles qui permettent d'obtenir un temps d'induction plus rapide que celui obtenu par l'inhalation d'une concentration à 1.5 pour cent de méthoxyflurane?

Nous en avons essayé trois:

1. L'inhalation d'une concentration supérieure à 1.5 pour cent pouvant atteindre un maximum de 3.3 pour cent $\left(20^{\circ} \mathrm{C}\right.$.), en prévoyant toujours un système de vaporisation permettant le passage d'un débit gazeux important (Verni-trol 3L).

2. Création d'une hyperventilation artificielle par prise de contrôle ou assistance de la respiration du patient.

Ces deux façons de procéder permettent sûrement de fournir une plus grande quantité de molécules gazeuses anesthésiques à la barrière alvéolo-capillaire et accélèrent sans aucun doute la création des coefficients sang/cerveau capables d'induire le sommeil. De fait, en utilisant la même prémédication et le méthoxyflurane comme seul agent anesthésique, il fut possible de ramener le temps d'inhalation (Penthrane à $3.3 \%$ nécessaire à une intubation sans relaxant) à une durée de quatre à cinq minutes. Des résultats à peu près semblables sont fournis par l'inhalation du méthoxyflurane à 1.5 pour cent sous hyperventilation. Il faut toutefois noter que ces deux procédés portent une atteinte plus rapide et plus sévère au système cardio-vasculaire que la technique décrite au début. Non pas que ces deux procédés soient à proscrire totalement mais ce sont des procédés qui diminuent la sécurité d'emploi presque inhérente à une substance telle que le méthoxyflurane. Ils peuvent être nécessaires à l'occasion mais leur emploi ne doit 
pas être routinier; les bons résultats et la rapidité suffisante du temps d'induction et la grande sécurité de l'emploi du méthoxyflurane à une concentration maxima de 1.5 pour cent semblent des arguments suffisants pour différer l'utilisation routinière de la ventilation forcée et de concentration supérieure à 1.5 pour cent.

3. Utilisation des relaxants musculaires: à cause de la brièveté de son action et de la tolérance surprenante de l'enfant vis-à-vis l'action dépolarisante de ce relaxant musculaire, la succinylcholine est la substance toute indiquée pour fournir les conditions que nous recherchons: rapidité et sécurité. Son emploi peut se faire par voie intraveineuse et intramusculaire à dose de $0.5-1.0 \mathrm{mg} . / \mathrm{kg}$. de poids suivant la voie d'administration. La succinylcholine est administrée dès l'apparition des premières respirations rythmiques soit environ trois minutes après le début de l'inhalation de méthoxyflurane: le patient est ainsi rapidement intubé et prêt pour la chirurgie dans un laps de temps plus que satisfaisant. Cette séquence méthoxyflurane-Succinylcholine avec respiration partiellement assistée à la reprise est une technique de tout premier choix, rapide et dénuée de manœuvre potentiellement dangereuse. Le système cardio-vasculaire est d'une stabilité remarquable et aucune arythmie cardiaque ne s'est manifestée. La rapidité d'action de cette association est comparable à celle de toute autre association de drogues servant à l'anesthésie moderne.

\section{Méthode en goutte à goutte}

Dix cas furent anesthésiés par la méthode du goutte à goutte: les plus jeunes sujets du groupe âgés de un et deux ans. La description de cette technique a été faite moulte fois depuis la venue du méthoxyflurane; simple, elle ne demande que le minimum de matériel et qu'une minime quantité de liquide anesthésique. Cette technique chez le tout jeune enfant amène la perte de conscience après un temps variable mais la moyenne de temps écoulé depuis le début de l'inhalation jusqu'à la perte apparente de conscience a été de trois minutes. La phase d'excitation est plus marquée qu'avec les méthodes décrites plus haut et s'accompagne de respiration retenue, de phonation et de mouvements souvent assez amples pour nécessiter une retention forcée.

L'évolution est graduelle et les signes classiques encore ici sont absents. Il faut noter qu'administré en goutte à goutte, le méthoxyflurane semble plus irritant pour les muqueuses respiratoires: toux occasionelle et surtout stimulation plus prononóe de glandes salivaires et des glandes muqueuses respiratoires.

Ce mode d'administration semble occasionner également une légère tendance à la tachycardie (cinq cas) et à l’apparition de cyanose aux lèvres et aux muqueuses unguéales. Ces deux contretemps sont immédiatement corrigés par l'introduction sous le masque d'un cathéter laissant fuser un léger débit $\mathrm{d}^{\prime} \mathrm{O}_{2}$ à une concentration de 100 pour cent.

Le relâchement musculaire est assez précose mais il faut un temps d'inhalation de huit à douze minutes pour obtenir des conditions satisfaisantes d'intubation sans utilisation de curare.

Les mêmes modifications respiratoires et circulatoires se rencontrent avec cette méthode que celles produites au cours de l'emploi des techniques décrites auparavant. 
La prescription d'une prémédication devient plus impérieuse dans le cas du goutte à goutte à cause de la tendance à l'hypersalivation et aussi à cause de la longuer du temps d'inhalation nécessaire à l'obtention du sommeil chirurgical. La belladone et ses dérivés par leur effet asséchant, contrecarre les inconvénients de l'hypersalivation et un barbiturique, employé même à dose conservatrice, est l'adjuvant nécessaire qui permettra d'atteindre plus facilement et plus rapidement le sommeil chirurgical.

Cette méthode d'anesthésie au méthoxyflurane par le goutte à goutte est assez facilement réalisable et sa sécurité est discutable même si l'on emploie un léger arrivage $\mathrm{d}^{\prime} \mathrm{O}_{2}$ sous le masque. Le temps nécessaire à une induction avec intubation sans relaxant soit huit à douze minutes, reste acceptable et se compare avantageusement avec le temps nécessaire à d'autres substances pour l'obtention d'un résultât semblable. Sans doute, le domaine didactique y a-t-il trouvé un nouvel auxiliaire.

Mais il demeure toutefois, que le manque de contrôle sur la respiration du patient amène une hypoxie hypercarbique, soit une raison suffisante pour ne pas utiliser cette méthode.

\section{RÉSUMÉ}

Le méthoxyflurane a été utilisé comme agent d'induction unique dans deux cent cinquante cas d'anesthésie chez des enfants de un à douze ans. Tous étaient de bons risques anesthésiques. Pour un grand nombre, l'admission à l'hôpital a en lieu matin même de l'intervention, et ceux-ci ont quitté le milieu hospitalier le jour même, aucun accident grave n'est survenu, aucune plainte des parents n'a été rapportée quant au comportement post-anesthésique. Les résultats ont été très bons et cet agent est utile et sûr comme agent d'induction en anesthésie pédiatrique.

\section{SUMMARY}

Methoxyflurane was used as the sole agent for induction of anaesthesia in 250 children whose ages ranged from one to twelve years. All were good anaesthetic risks. A great many of them were admitted on the morning of surgery and left the hospital the same day.

Different methods of administration were used: partial rebreathing without $\mathrm{CO}_{2}$ absorption, non-rebreathing, and the open drop technique. The first two techniques were very satisfactory, while the last one was considered dangerous because it produces hypercarbic hypoxia. No serious accidents occurred and there were no complaints from the parents regarding the postanaesthetic course. The results were very good, indicating that this compound is a useful and safe agent for the induction of anaesthesia in paediatrics.

\section{REMERCIEMENTS}

Nos remerciements vont à la compagnie pharmaceutique Abbott pour l'aide financière que ses représentants nous ont accordée. 


\section{REFERENCES}

1. Adriani, F., JR. Halogenated Anaesthetics. Clin. Anesth. (1963).

2. Déry, R.; Pelletier, J.; \& JACQues, A. Comparaison des effets respiratoires et metaboliques du mélange azéotrope fluothane-éther et du méthoxyflurane. Canad. Anaesth. Soc. J. 11: 410 (1964).

3. PEREY, B. \& DufF, J. Evaluation clinique et traitement du choc basé sur un diagnostic hémodynamique. 